\section{Diabetes Mellitus tipo 2: desafíos para los modelos de cuidados crónicos en Chile}

\author{
ALEXIS GONZÁLEZ-BURBOA ${ }^{1,3,4, \mathrm{a}, \mathrm{d}}$, ALDO VERA-CALZARETTA ${ }^{1,3,4, \mathrm{~b}, \mathrm{c}}$, \\ PATRICIA VILLASECA-SILVA ${ }^{3}$, HANS MÜLLER-ORTIZ ${ }^{2,4}$
}

\section{Type 2 diabetes mellitus as a challenge for chronic disease care models in Chile}

The increase in chronic noncommunicable diseases is one of the main concerns worldwide. Therefore, it is pertinent to ask how care system organizations responded to the requirements currently being posed by chronic diseases, specifically type 2 diabetes mellitus (T2DM). In the world, different models have been developed considering elements that transcend the biological stability of the disease. Chile has the great challenge of exploring new models that emphasize the promotion and prevention of health rather than the management of the disease. The impact of variables such as health literacy, self-efficacy and subjective well-being on T2DM should be explored. In addition, it is necessary to validate and implement guidelines and protocols of care that incorporate the aforementioned variables.

(Rev Med Chile 2019; 147: 361-366)

Key words: Diabetes Mellitus, Type 2; Health Services; Noncommunicable Diseases.

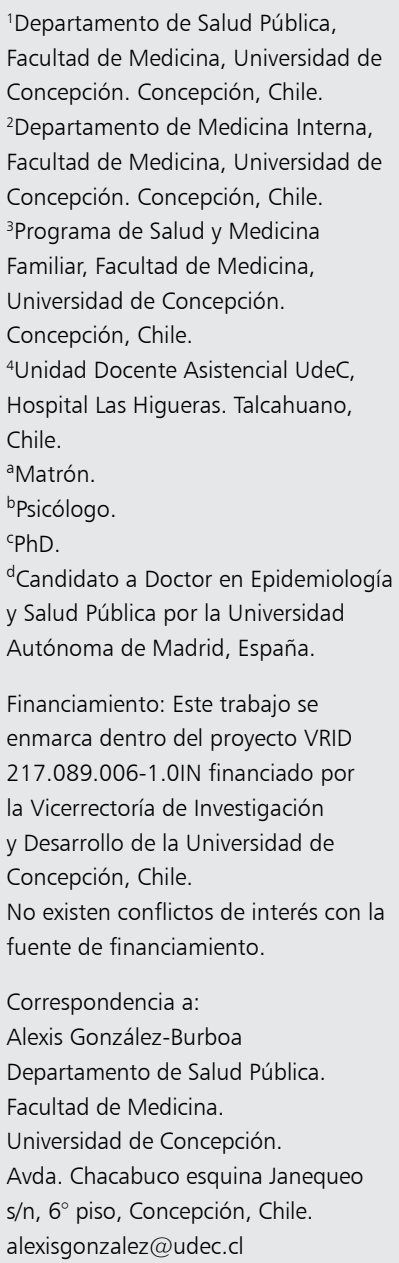

Financiamiento: Este trabajo se enmarca dentro del proyecto VRID 217.089.006-1.0IN financiado por la Vicerrectoría de Investigación y Desarrollo de la Universidad de Concepción, Chile.

No existen conflictos de interés con la fuente de financiamiento.

Correspondencia a:

Alexis González-Burboa

Departamento de Salud Pública.

Facultad de Medicina.

Universidad de Concepción. Avda. Chacabuco esquina Janequeo s/n, $6^{\circ}$ piso, Concepción, Chile. alexisgonzalez@udec.cl

$\mathrm{E}$ 1 propósito de este trabajo es reflexionar en torno a los desafíos del cuidado de la Diabetes Mellitus tipo 2 (DM2) en Chile a la luz de algunos modelos de cuidado de la cronicidad desarrollados en el mundo. Para esto, a continuación se presentan cuatro ejes temáticos. El primero de ellos, entrega una breve introducción a la situación epidemiológica de las Enfermedades Crónicas No Transmisibles (ECNT) y la DM2 a nivel internacional y local. El segundo, muestra algunos de los modelos teóricos para el abordaje del cuidado de pacientes crónicos desarrollados en las últimas décadas. El tercero, describe la evolución del cuidado de la DM2 en Chile, y finalmente, el cuarto, señala algunos aspectos a considerar frente al desafío del cuidado de la DM2 en nuestro país.

\section{Enfermedades crónicas no transmisibles y Diabetes Mellitus tipo 2: un problema en crecimiento}

El aumento de las ECNT es una de las principales preocupaciones a nivel mundial. Según datos internacionales, el año 2016, de las 57 millones de muertes ocurridas en el mundo, 71\% (41 millones) fueron causadas por $\mathrm{ECNT}^{1}$, alcanzando estas 15\% en la Región de las Américas ${ }^{2}$. En este sentido, se ha señalado que en los países de bajos y medianos ingresos se presentan las tasas más altas de mortalidad de personas menores de 70 años ${ }^{3}$, llegando incluso a doblar la cantidad de muertes en comparación a los países de altos ingresos (21\% y $23 \%$ respectivamente, versus $12 \%$ ). Cabe destacar 
que, independientemente de la zona geográfica de procedencia, la probabilidad de morir producto de una ECNT es mayor en el sexo masculino ${ }^{2}$.

En relación a las causas de muerte específicas por ECNT, una de las más relevantes es la Diabetes Mellitus, cuya forma más frecuente es el tipo $2-\mathrm{DM} 2-{ }^{4}$,y que por sí sola representa $3 \%$ de la mortalidad mundial total ${ }^{1}$. Al respecto, en el año 2017 la prevalencia de personas con diabetes entre 20 a 79 años ascendió a 8,8\%, estimándose que al año 2045 esta aumentará a 9,9\% ${ }^{5}$. Por otra parte, al considerar el gasto sanitario asociado a la enfermedad, se estima que las personas con diabetes gastan el doble de recursos (USD) en comparación con aquellas personas que no tienen la enfermedad ${ }^{5}$.

¿Qué pasa en Chile? Actualmente, la esperanza de vida en nuestro país es de 79,05 años, teniendo el sexo femenino aproximadamente 5 años más de vida (81,69 años versus 76,52 años). Según datos de la última Encuesta Nacional de Salud ${ }^{6}$, en nuestro país $40,1 \%$ de la población tiene síndrome metabólico, encontrándose diferencias significativas entre las personas de 45 o más años y las personas de menor edad ( $\mathrm{p}<0,001)$. Además, los grupos de personas entre 45 y 64 años y junto con aquellas de 65 o más años (36,4\% y 65,6\% respectivamente), presentan un alto riesgo cardiovascular. Al revisar las causas de muerte en nuestro país, $85 \%$ corresponde a ECNT, de las cuales $5 \%$ son ocasionadas por la DM2 ${ }^{2}$. Según datos de la Federación Internacional de la Diabetes ${ }^{5}$, al año 2017, en Chile la prevalencia de personas con diabetes entre 20 y 79 años ascendió a $8,5 \%$ de la población.

Frente a la situación de las ECNT, y en específico de la DM2en Chile y el mundo, es pertinente preguntarse: ¿Cómo se ha respondido desde la perspectiva de la organización de los sistemas de cuidado a los requerimientos que está planteando actualmente la cronicidad??

\section{Modelos para el abordaje del cuidado de la cronicidad}

¿Qué es un modelo de cuidado? Kuerten et $\mathrm{al}^{8}$, lo definen como: "[...] estructura del conocimiento... formada por un marco de referencia (o marco teórico) y una metodología. Posee un nivel de abstracción inferior al de la teoría, porque puede ser derivado de una o más teorías, y presenta, además de un aporte filosófico y teórico, una posibilidad de planeamiento y sistematización del cuidado....nos remite a la construcción de un conocimiento que favorece un cuidar diferenciado y específico, que auxilia el cuidado prestado al ser humano [...]". En esta lógica, existen distintas formulaciones que han conceptualizado aspectos del cuidado que consideran elementos que trascienden solo a la estabilidad biológica de la enfermedad.

$\mathrm{Al}$ respecto, a finales del siglo veinte Edward Wagner ${ }^{9}$ propuso un Modelo de Cuidados Crónicos (Chronic Care Model-CCM-), específicamente dirigido a personas con ECNT, que ha servido de base a otros modelos de cuidado. Este plantea que los cuidados producidos por la interacción entre los proveedores de servicios y los pacientes que obtienen mejores resultados, son observables en sistemas que: a) entregan servicios con incentivos y procesos bien desarrollados; b) apoyan la autogestión de los pacientes; c) reconocen el rol del equipo de salud y los sistemas de prácticas en el conocimiento de las necesidades de los enfermos crónicos; d) desarrollan e implementan guías de práctica basadas en la evidencia, y e) mejoran los sistemas de información, es decir, el registro, seguimiento y retroalimentación sobre el desempeño. Como una propuesta de mejora del MCC, Barr et $\mathrm{al}^{10}$ desarrollaron el Modelo Expandido de Cuidados Crónicos (Expanded Chronic Care Model-ECCM-), el cual agrega la perspectiva de la promoción de la salud al cuidar, no solo reducir el impacto de las ECNT en la personas, sino también apoyar a las mismas y a sus comunidades para estar saludables. El ECCM presta especial atención al impacto que los determinantes sociales pueden tener sobre la salud de los pacientes crónicos.

Otra expansión del MCC que fue planteada por la Organización Mundial de la Salud, es conocida como Marco para el Cuidado Innovador de las Condiciones Crónicas (Innovative Care for Chronic Conditions Framework-ICCCF-) ${ }^{11}$. Este modelo plantea una interesante mirada desde la perspectiva del apoyo a las necesidades de las personas y sus familias, integrando los niveles macro (políticas públicas), meso (organización de los cuidados de salud y las comunidades) y micro (interacción con los pacientes) en la gestión o prevención de las enfermedades crónicas. En este sentido, la innovación se plantea en la integración de estos niveles y en la re-conceptualización de lo que se entiende por enfermedad crónica, reconociendo que la calidad de vida de los pacientes y 
sus familias es un resultado importante y, además, que estos tienen un importante rol sobre la misma, considerándolos "productores de salud".

Los modelos previamente señalados permiten tener una comprensión teórica y metodológica de cómo las políticas y sistemas de salud pueden responder a las necesidades de las personas que tienen ECNT. No obstante, en la gestión del cuidado propiamente tal, uno de los modelos más reconocidos es el planteado por la aseguradora Kaiser Permanente -KP_ ${ }^{12,13}$, basado en la caracterización de la población que recibe servicios de salud y la estratificación de dicha población en función de la intensidad del uso de recursos. KP persigue mejorar la salud de las personas con ECNT reduciendo el uso de recursos en el nivel de atención más costo-efectivo. En esta misma línea, un complemento a este modelo es el King's Fund -KF- del Reino Unido, que agrega la perspectiva socio-sanitaria en cada uno de los niveles de estratificación planteados por KP. Desde el punto de vista social, el KF incorpora la asistencia domiciliaria a personas con dependencia, el soporte a los cuidadores y la inversión en voluntariado. En relación a la perspectiva sanitaria, se pone el acento en la prevención de las hospitalizaciones, el diagnóstico precoz y la promoción de los estilos de vida saludables ${ }^{14}$.

Dado que una de las características de las ECNT es que no suelen presentarse de forma única, se ha dado paso al desarrollo de algunos modelos de cuidados para apoyar a personas que presentan multimorbilidad ${ }^{15,16}$. En particular, cabe destacar el caso del proyecto denominado Modelos Sustentables de Cuidados Crónicos Integrados para Multimorbilidad: Entrega, Financiamiento y Desempeño (Sustainable Integrated Chronic Care Models for Multi-Morbidity: Delivery, Financing, and Performance-SELFIE-) ${ }^{17}$. SELFIE es el resultado del trabajo de un consorcio integrado por Holanda, Austria, Croacia, Alemania, Hungría, Noruega, España y el Reino Unido, que apunta a la mejora de los cuidados mediante la propuesta de programas integrados basados en la persona, económicamente sustentables y basados en la evidencia. Para ello, SELFIE plantea un marco conceptual que sitúa al individuo con multimorbilidad y su entorno en el centro. Luego, reconoce una serie de elementos relacionados con el cuidado integrado de la multimorbilidad (por ejemplo, autogestión, toma de decisiones compartidas, in- vestigación innovadora, regulación del mercado, equidad y acceso, entre otros) y los sitúa en tres niveles (micro, meso y macro). Finalmente, cada uno de estos niveles, a la vez, se agrupa en seis grandes dimensiones a fin de monitorear la implementación del modelo: a) entrega de servicios; b) liderazgo y gobernanza; c) fuerza de trabajo; d) financiamiento; e) tecnologías y productos médicos, y f) información e investigación.

\section{Cuidado de la diabetes en Chile}

$\mathrm{Al}$ analizar el caso del cuidado de la DM2 en Chile, a través de su Estrategia Nacional de Salud ${ }^{6}$, el país se ha propuesto alcanzar al año 2020 una cobertura de control de personas con DM2 de 35,8\%. Para el logro de esta meta la principal estrategia existente en el país corresponde al Programa de Salud Cardiovascular $-\mathrm{PCV}-{ }^{18}$, cuyo objetivo es reducir la incidencia de eventos cardiovasculares y aumentar el control de las personas con algún evento ya producido. Cabe destacar que este programa sumó el año 2009, a fin de orientar el manejo clínico y seguimiento de pacientes con riesgo cardiovascular, la incorporación del modelo de atención con enfoque de riesgo ${ }^{19}$. Por otra parte, la DM2 se encuentra incluida dentro de las Garantías Explicitas en Salud-GES-20 vigentes, las cuales consideran como una de sus prestaciones la inclusión del Examen de Medicina Preventiva para la pesquisa de nuevos $\operatorname{casos}^{21}$. Sumado a lo anterior, el año 2017 se actualizó el manejo farmacológico de los pacientes con DM2 mediante la "Guía de Práctica Clínica para el Tratamiento Farmacológico de la Diabetes Mellitus tipo 2"22.

Las estrategias diseñadas para responder a las demandas del cuidado de las personas con DM2 mencionadas previamente, cuyo foco principal es el manejo clínico, requieren insertarse en la estructura prestadora de servicios del sistema de salud para ser llevadas a cabo. $\mathrm{Al}$ respecto, Chile posee un sistema que reconoce como puerta de entrada a la atención primaria-APS-23,24, nivel de la red donde se espera producir el mayor impacto en el cuidado de las personas con ECNT.

En las últimas décadas la APS chilena ha ido transitando desde un enfoque biomédico a uno que permite considerar a la persona, su familia y su comunidad como actores clave en el cuidado de su salud ${ }^{25-29}$. Al respecto, se ha venido instalando 
progresivamente un Modelo de Atención Integral de Salud Familiar y Comunitaria -MAISFC- ${ }^{29}$ que sitúa a los equipos de salud en una constante relación con las personas, sus familias y las comunidades de un territorio dado, empoderándolas para tomar decisiones relacionadas con el cuidado de su salud dentro de un entorno heterogéneo y complejo. En este modelo, el sistema sociosanitario se ordena en torno a las necesidades de los individuos a fin de que estos sean actores movilizados en lo que respecta al cuidado de su salud y, en específico, plantea que la continuidad del cuidado, la integralidad y el tener como centro a la persona y su familia, son principios irrenunciables. El MAISFC monitorea su implementación mediante un proceso de certificación que da cuenta de la presencia de estos tres principios en nueve ejes estratégicos: a) promoción de la salud; b) prevención en salud; c) enfoque familiar; d) participación de la comunidad en salud; e) calidad; f) intersectorialidad y territorialidad; $\mathrm{g}$ ) gestión del desarrollo de las personas y de la organización; h) tecnología, e i) centro en la atención abierta. Es importante señalar que el abordaje de la DM2 es una de las preocupaciones que ha considerado este modelo en actividades, tales como, consejerías familiares, planes de cuidado del cuidador, coordinación entre el sector primario, secundario y terciario para el seguimiento de la atención, y planes de prevención de la progresión de la enfermedad, entre otros ${ }^{30}$.

Pese a los esfuerzos realizados, en Chile no hay actualmente modelos "completos" para enfrentar las ECNT, y particularmente la DM2, quedando aún mucho trabajo por hacer.

Así, al considerar sus constantes innovaciones, la APS ofrece las mayores posibilidades de introducir nuevas formas de abordar el cuidado de las ECNT, específicamente de la DM2. Una de esas formas innovadoras se propuso el año 2015 a través de las directrices para la implementación del "Modelo de Atención para Personas con Enfermedades Crónicas" 31 que, tomando cada uno de los componentes propuestos por el modelo de Edward Wagner ${ }^{9}$, planteó una serie herramientas e instrumentos de medición para el trabajo de los equipos de los servicios de salud del país. No obstante, las posibilidades que presenta este modelo, han existido una serie de limitaciones en la implementación del mismo, tales como, la evaluación de la pertinencia cultural, la planificación del recurso humano necesario, la planificación de la cartera de servicios en base a las necesidades de la población objetivo, el desconocimiento del modelo por parte de los equipos de salud, la hegemonía aun reinante del modelo biomédico por sobre otros modelos, todos aspectos que son tema de otra reflexión.

\section{Consideraciones finales}

Los sistemas de salud en el mundo hoy enfrentan el gran desafío de replantearse la forma en que se está dando respuesta a las crecientes demandas que la transición demográfica y el cambio en el perfil epidemiológico de la población están generando. Las ECNT, y en especial la DM2, están a la base de una gran cantidad de muertes en el mundo y Chile no está exento de esta realidad. En este sentido, una de las condiciones que complejiza el cuidado de las personas que tienen DM2 está dada por, la mayor parte de las veces, la existencia de pluripatología. Esta condición, genera necesidades y expectativas de cuidado de un mayor nivel de complejidad, las cuales, no solo consideran el manejo médico de la enfermedad, sino también, la preocupación por aspectos psicosociales que generan gran impacto en la calidad de vida y en el propio cuidado de las personas.

Otro aspecto a considerar es que los esfuerzos en el cuidado deben necesariamente estar dirigidos a prevenir el avance de la DM2 hacia una condición que genere dependencia. La dependencia, junto con mellar la calidad de vida de las personas, afecta el entorno familiar y genera la sobrecarga de quienes se dedican al cuidado. Así, la figura del cuidador(a) no se encuentra lo suficientemente considerada en el abordaje que plantean las estrategias implementadas en nuestro país y constituye uno de los aspectos que deben ser mejorados.

Pese a los desarrollos que han experimentado los modelos de cuidado en el mundo, y a las distintas estrategias que se han implementado en Chile, una de las tareas aún pendientes es transitar desde un sistema de cuidados estandarizados hacia uno que permita instalar la práctica de cuidados centrados en la persona. No obstante los intentos por introducir un cambio en la forma de concebir la organización de los cuidados, es vital reconocer que se sigue poniendo énfasis en el abordaje basado en el continuo salud-enfermedad, pero no en aspectos que muchas de las propuestas teóricas presentadas en este trabajo han mostrado. Pese al 
intento por incorporar la propuesta del MCC en el sistema de salud chileno, aún no se ha logrado una implementación que permita incorporar cada uno de sus componentes.

Un gran desafío es explorar nuevos modelos que enfaticen en la promoción y prevención de la salud más que en el manejo de la enfermedad. En este sentido, estudiar el impacto que variables como la alfabetización en salud, la autoeficacia y el bienestar subjetivo tienen en el cuidado de la DM2 es una oportunidad a explorar. Además, es necesario avanzar en la validación e implementación de guías y protocolos de atención que incorporen variables como las mencionadas previamente en el manejo de las personas con DM2.

\section{Referencias}

1. World Health Organization. World health statistics 2018: monitoring health for the SDGs, sustainable development goals. Geneva: World Health Organization; 2018.

2. World Health Organization. Noncommunicable diseases country profiles 2018. Geneva: World Health Organization; 2018.

3. World Health Organization. Global status report on noncommunicable diseases 2014: attaining the nine global noncommunicable diseases targets; a shared responsability. Global status report on noncommunicable diseases 2014: attaining the nine global noncommunicable diseases targets; a shared responsibility. Geneva: World Health Organization; 2014.

4. Federación Internacional de la Diabetes. Atlas de la Diabetes de la FID. [Internet]. Sétima edición. International Diabetes Federation; 2015. [Citado el 14 de enero de 2019]. Disponible en: https://www.fundaciondiabetes. org/upload/publicaciones_ficheros/95/IDF_Atlas_2015_ SP_WEB_oct2016.pdf.

5. Federación Internacional de la Diabetes. Atlas de la Diabetes de la FID. [Internet]. Octava edición. International Diabetes Federation; 2017. [Citado el 14 de enero de 2019]. Disponible en: http://diabetesatlas.org/ IDF_Diabetes_Atlas_8e_interactive_ES/

6. Ministerio de Salud. Encuesta Nacional de Salud 2016-2017: segunda entrega de resultados. [Internet]. Santiago: Ministerio de Salud; 2018. [Citado el 14 de enero de 2019]. Disponible en: https://www.minsal.cl/ wp-content/uploads/2018/01/2-Resultados-ENS_MINSAL_31_01_2018.pdf.

7. Carrillo E, Cervera M, Gil V, Rueda Y. Claves para la construcción del espacio sociosanitario en España. Barcelona: Antares Consulting; 2010.

8. Kuerten P, Lenise do Prado M. Modelo de Cuidado: ¿Qué es y cómo elaborarlo? Index de Enfermería 2008; 17 (2): 128-32.

9. Wagner EH. Chronic disease management: what will it take to improve care for chronic illness? Eff Clin Pract 1998; 1 (1): 2-4.

10. Barr VJ, Robinson S, Marin-Link B, Underhill L, Dotts A, Ravensdale D, et al. The expanded Chronic Care Model: an integration of concepts and strategies from population health promotion and the Chronic Care Model. Hosp Q 2003; 7 (1): 73-82.

11. Pruitt S, Epping-Jordan J. Innovative care for chronic conditions: building blocks for action: global report. Geneva: World Health Organization; 2002.

12. Feachem RG, Dixon J, Berwick DM, Enthoven AC, Sekhri NK, White KL. Getting more for their dollar: a comparison of the NHS with California's Kaiser Permanente Commentary: Funding is not the only factorCommentary: Same price, better care Commentary: Competition made them do it. BMJ 2002; 324: 135-43.

13. Solinís RN. Buenas prácticas en gestión sanitaria: el caso Kaiser Permanente. Rev Adm Sanit 2007; 5(2): 283-92.

14. Humphries R. Social Care Funding and NHS: An Impending Crisis? London: King's Fund; 2011.

15. Palmer K, Marengoni A, Forjaz MJ, Jureviciene E, Laatikainen T, Mammarella F, et al. Multimorbidity care model: Recommendations from the consensus meeting of the Joint Action on Chronic Diseases and Promoting Healthy Ageing across the Life Cycle (JA-CHRODIS). Health Policy 2018; 122 (1): 4-11.

16. National Institute for Clinical Excellence. Multimorbidity: clinical assessment and management. United Kingdom: NICE; 2016.

17. Leijten FR, Struckmann V, van Ginneken E, Czypionka T, Kraus M, Reiss M, et al. The SELFIE framework for integrated care for multi-morbidity. Health Policy 2018; 122 (1): 12-22.

18. Ministerio de Salud, Gobierno de Chile. Orientación Técnica: Programa de Salud Cardiovascular 2017. Santiago: Ministerio de Salud; 2017.

19. Ministerio de Salud, Gobierno de Chile. Implementación del enfoque de riesgo en el programa de salud cardiovascular. Santiago: Ministerio de Salud; 2009.

20. Ministerio de Salud, Gobierno de Chile. Guía clínica: Diabetes Mellitus tipo 2. Santiago: Ministerio de Salud; 2010.

21. Ministerio de Salud, Gobierno de Chile. Guía clínica: 
Examen de Medicina Preventiva. Segunda edición. Santiago: Ministerio de Salud; 2013.

22. Ministerio de Salud, Gobierno de Chile. Guía de Práctica Clínica: Tratamiento Farmacológico de la Diabetes Mellitus tipo 2 2016-2017. Santiago: Ministerio de Salud; 2017.

23. Benavides P, Castro R, Jones I. Sistema público de salud, situación actual y proyecciones fiscales 2013-2050. Santiago: Dirección de Presupuestos del Ministerio de Hacienda; 2013.

24. Ministerio de Salud, Gobierno de Chile. Orientaciones para la planificación y programación en red 2019. Santiago: Ministerio de Salud; 2019.

25. Ministerio de Salud, Gobierno de Chile. De consultorio a centro de salud. Primera edición. Santiago: Ministerio de Salud; 1993.

26. Ministerio de Salud, Gobierno de Chile. Modelo de atención integral en salud. Santiago: Ministerio de Salud; 2005.

27. Ministerio de Salud, Gobierno de Chile. En el camino a
Centro de Salud Familiar. Santiago: Ministerio de Salud; 2008.

28. Ministerio de Salud, Gobierno de Chile. Orientaciones para la implementación del modelo de atención integral de salud familiar y comunitaria. Santiago: Ministerio de Salud; 2013.

29. Ministerio de Salud, Gobierno de Chile. Modelo de atención integral con enfoque familiar y comunitario en establecimientos de la red de atención de salud. Santiago: Ministerio de Salud; 2008.

30. Ministerio de Salud, Gobierno de Chile. Instrumento para la evaluación y certificación de desarrollo en el modelo de atención integral de salud familiar y comunitaria en establecimientos de atención primaria. Santiago: Ministerio de Salud; 2015.

31. Ministerio de Salud, Gobierno de Chile. Modelo de atención para personas con enfermedades crónicas: directrices para la implementación. Santiago: Ministerio de Salud; 2015. 